\title{
Identifying Fe I Levels from Stellar Spectra
}

\author{
Ruth C. Peterson
}

SETI Institute, 189 N Bernardo Ave, Mountain View, CA 94043

\begin{abstract}
The spectrum of the Fe I atom is critical to many areas of astrophysics and beyond. Measurements of the energies of its high-lying levels remain seriously incomplete. Here we update the work by Peterson \& Kurucz (2015) to identify such levels using new and archival highresolution absorption-line UV and optical spectra of stars whose warm temperatures favor Fe I lines. Our GO-14161 Hubble program will obtain UV spectra for four metal-poor turnoff stars, archive them at ASTRAL, and draw from them the new identifications of $250-500 \mathrm{Fe}$ I levels.
\end{abstract}

Keywords. atomic data, techniques: spectroscopic, stars: Population II, ultraviolet: stars

Extensive, reliable data for the iron atom are especially critical to astrophysics, owing to the rich spectrum and the high cosmic abundance of iron. Yet the current laboratory data for neutral iron remain woefully incomplete. The result is that fluxes of old stars cannot yet be computed from first principles. As seen in Figure 1 of Peterson \& Kurucz (2015), the presence of large numbers of unidentified Fe I lines near $2600 \AA$ causes calculated fluxes of solar-temperature stars to fall increasingly above observed fluxes as metallicity increases. The excess of predicted flux reaches a factor of three at solar metallicity.

Peterson \& Kurucz (2015) undertook to identify these lines from archived UV spectra of the turnoff stars themselves, over a wide metallicity range. They found the upper energies for $66 \mathrm{Fe}$ I levels. Many are of higher excitation than achieved in laboratory experiments; several exceed the Fe I ionization energy. From these levels over 2000 potentially detectable Fe I lines are identified from $1600 \AA$ to $5.4 \mu, 1 / 3$ of which are in the infrared, and astrophysical $g f$ values are listed where meaningful. Many promising level identifications remain unconfirmed, as their lines fall mostly in unobserved UV regions.

HST GO-14161 will remedy this. Peterson, Kurucz, \& Ayres will obtain STIS E230H high-resolution spectra to provide complete spectral coverage throughout the near UV for four halo metal-poor turnoff stars, HD 84937, HD 94028, HD 140283, and HD 211998. These fill in gaps in the archival coverage of the latter three stars, and provide the first E230H template of HD 84937, a truly metal-poor turnoff star. Ayres will reduce these spectra and incorporate them into the ASTRAL database (Carpenter \& Ayres 2015).

From these spectra, Peterson \& Kurucz will extend the Fe I identifications to hundreds more levels with even higher energies and weaker lines. How many new levels will emerge will depend on the effort funded: the spectra could reveal 500 new levels, but the budget may allow only half this number. The results will significantly improve determinations of the abundances of trace elements for metal-poor stars and of iron for dust-obscured red giants, and provide better metallicity and age determinations from spectra of old stellar systems, notably at high redshifts where the UV is shifted into the optical.

\section{References}

Carpenter, K. G. \& Ayres, T. R. 2015, in 18th Cambridge Workshop on Cool Stars, Stellar Systems, and the Sun, eds. G. van Belle and H. C. Harris, p. 1041

Peterson, R. C. \& Kurucz, R. L. 2015, ApJS, 216, 1 